\title{
Amniotic Membrane in Ophthalmology
}

\section{Prateeksha Sharma*}

Department of Ophthalmology, Guru Nanak Eye Centre, Delhi, India

*Corresponding Author: Prateeksha Sharma, Department of Ophthalmology, Guru Nanak Eye Centre, Delhi, India.
Received: April 13, 2020

Published: May 22, 2020

(C) All rights are reserved by Prateeksha

Sharma.

\begin{abstract}
The amniotic membrane is the inner most layer of the three fetal membranes lying deep to chorion. It is translucent and avascular in nature. It is derived from fetal ectoderm by cavitation within the fetal knot. It is composed of three layers: epithelium, basement membrane and stromal [1]. The structural integrity, transparency and elasticity makes the amniotic membrane most widely accepted tissue for ocular surface reconstruction.

Keywords: Amniotic Membrane; Fetal; Eye
\end{abstract}

\section{Introduction}

Various researches have shown that amniotic membrane has antimicrobial properties which help in reducing the risks of postoperative infection [2]. It is antifibrotic [3] in nature as its stroma contains fetal hyaluronic acid which inhibits fibroblast growth which also prevents scarring. It decreases expression of cytokines thus reduce inflammation. It acts as a bandage contact lens allowing conjunctival and corneal epithelization to occur under its cover. It promotes cell migration and growth [4]. Amniotic membrane produce various growth factors such as basic fibroblast growth factor, transforming growth factor $\beta$, hepatocyte growth factor that stimulate epithelization [5]. It is anti-angiogenic in nature. It also has additional bacteriostatic, wound protection, pain reduction activity. It does not express HLA-A, B, or DR antigen and hence immunological rejection after its transplantation does not occur [6].

\section{Procurement of amniotic membrane}

Amniotic membrane can be easily obtained and its availability is nearly unlimited. Tissue can be preserved at $-8^{\circ} \mathrm{C}$ for several months allowing sufficient time to plan for surgery. It is obtained under sterile conditions after elective caesarean delivery from a seronegative donor. Serum sample from all donors are tested for anti-HIV-1 and 2, hepatitis B, surface antigen anti-hepatitis C virus, syphilis. Serologic tests on the maternal donor must be done both at the time of procurement of the donor tissue and again on six months to eliminate the slightest risk of disease transmission. In lamellar flow hood, the placenta is first washed free of blood clots with balanced saline solution containing $50 \mu \mathrm{g} / \mathrm{ml}$ of penicillin, 50 $\mu \mathrm{g} / \mathrm{ml}$ of streptomycin, $100 \mu \mathrm{g} / \mathrm{ml}$ of neomycin and $2.5 \mu \mathrm{g} / \mathrm{ml}$ of amphotericin B. The inner amniotic membrane is separated from the rest of the chorion by blunt dissection (through the potential spaces between these two tissues). The membrane is then flattened onto a nitrocellulose paper, with the epithelium/basement membrane surface up.

Types of amniotic membrane: Mainly two main types are available

1. Cryopreserved amniotic membrane: The tissue is frozen at $-80^{\circ} \mathrm{C}$ in a sterile vial containing Dulbecco's modified Eagle's medium and glycerol at a ratio of 1:1 (vol/vol). It is defrosted immediately before use by warming the container to room temperature for 10 minutes [7]. ProKera (Bio Tissue) is a cryopreserved form where membrane is secured around a polycarbonate ring or an elastomeric band.

2. Dehydrated amniotic membrane: Membrane is preserved at low temperature using vacuum to retain devitalized cellular components. It can be kept at room temperature and needs to be rehydrated before use. AmbioDisk (IOP Ophthalmics) is a dehydrated amniotic membrane commercially available.

\section{Clinical applications}

The use of amniotic membranes in ocular surgery was first suggested by de Rotth [8] who examined its role in the management of ocular surface damage. 
There are three basis technique of using amniotic membrane:

- Graft technique: When it is used with the expectation that it will became epithelized and merge into the host tissue.

- Patch technique: When the amniotic membrane is used to cover an area of the ocular surface and is eventually removed or falls off, it is referred to as a "patch". When used as a patch it is expected that epithelialization will occur beneath the membrane, with the membrane acting as a bandage.

- $\quad$ Filling-in technique: The entire depth of an ulcer crater if filled with AM pieces.

The suture material used in conjunction with the amniotic membrane is usually 10-0 nylon, 8 to $10-0$ vicryl or prolene. The sutures may be interrupted, running or mattress in type. The membrane is always sutured to the ocular surface with its epithelial side up and the mesenchymal surface in contact with the eye, to facilitate adherence of the membrane to the ocular surface. For this reason, it is important to be able to distinguish its two surfaces. After spreading the membrane on the ocular surface, sponge is touched to one surface of the membrane a fine strand of "vitreouslike" substance can usually be drawn up from the mesenchymal but not from the epithelial (basement membrane). The amniotic membrane is spread on to surface of the eye and cut to appropriate size and shape, keeping the final piece slightly larger than the size of the defect to be covered.

\section{Indications of AMT in ocular surgery}

1. Conjunctival surface reconstruction

2. Pterygium surgery

3. Chemical burns

4. Cicatrizing conjunctivitis

5. Ocular surface squamous neoplasia (OSSN)

6. Leaking blebs

7. Filtering surgery Symblepharon release

8. Fornix formation

9. Socket reconstruction

10. Conjunctivochalasis

11. Entropion correction

12. Corneal surface reconstruction

13. Persistent epithelial defects.
14. Non-healing stromal ulcers

15. Partial Limbal stem cell deficiency

16. Total Limbal stem cell deficiency

17. Bullous keratopathy

18. Band keratopathy.

Complications: Several problems can occur after amniotic membrane transplantation. The amniotic membrane may disintegrate before epithelisation, in some cases within 2 weeks after transplantation. Necrosis of the amniotic membrane may occur. The amniotic membrane does not remain attached to the ocular surface if the mesenchymal surface is not facing the host. Suture granuloma, persistent inflammation are other drawbacks. There can be loss of membrane, either by degradation or by cheese wiring of the sutures, in the immediate postoperative period. Another less significant undesirable effect is the residual subepithelial membrane that persists in some cases. Accumulation of blood (hematoma formation) under the membrane can occur in the immediate postoperative period. Transmission of infections can also occur so testing for infections must be done.

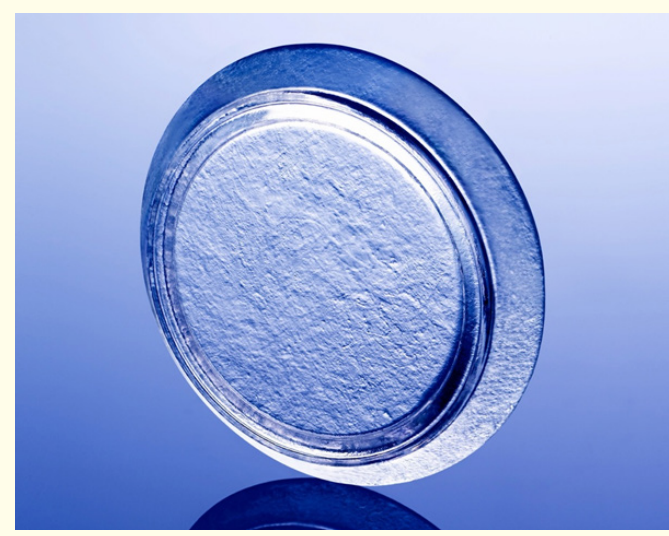

Figure 1: ProKera (Bio tissue).

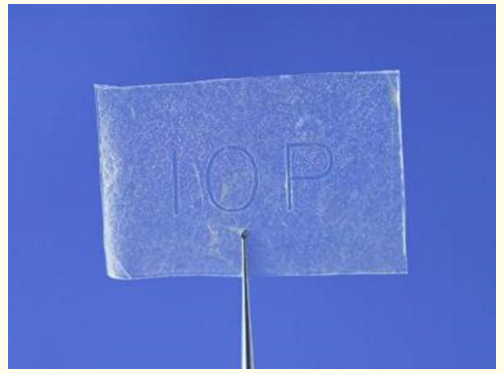

Figure 2: AmbioDisk with IOP mark on epithelial side. 
Recent advances: While Amniotic membrane has traditionally been transplanted using sutures. Adhesives like fibrin glue has converted amniotic membrane transplantation into a sutureless surgery. Cryopreserved ProKera is less invasive alternative where superior ring is placed under the upper eyelid while patient looking down and then having patient looking up, the remainder ring is placed onto the eye. The membrane is supplied in such a way that stromal side always faces the ocular surface. This can be done under topic anaesthesia as an office procedure. Another in office use alternative is dehydrated AmbioDisk where the membrane dis is placed on ocular surface with "IOP" watermark facing physician thus ensuring the contact of stromal surface with ocular surface. Few drops of sterile saline are put to rehydrate the membrane. Bandage contact lens is placed over it.

\section{Bibliography}

1. Danforth DN and Hull RW. "The microscopic anatomy of the fetal membranes with particular reference to the detailed structure of the amnion". American Journal of Obstetrics and Gynecology 75.3 (1958): 536-550.

2. Talmi YP., et al. "Antibacterial properties of human amniotic membranes". Placenta 12.3 (1991): 285-288.

3. Akle CA., et al. "Immunogenicity of human amniotic epithelial cells after transplantation into volunteers". Lancet 2.8254 (1981): 1003-1005.

4. Shimazaki J., et al. "Transplantation of amniotic membrane and limbal autograft for patients with recurrent pterygium associated with symblepharon". British Journal of Ophthalmology 82.3 (1998): 235-234.

5. Sato H., et al. "Role of growth factors for ocular surface reconstruction after amniotic membrane transplantation". Investigative Ophthalmology and Visual Science 39 (1998): S428.

6. Adinofli M., et al. "Expression of HLA antigens, â2-microglobulin and enzymes by human amniotic membrane". Nature 295.5847 (1982): 325-327.

7. Tseng SCG., et al. "Amniotic membrane transplantation for conjunctival surface reconstruction". American Journal of $\mathrm{Ob}$ stetrics and Gynecology 124.6 (1997): 765-774.

8. De Rotth A. "Plastic repair of conjunctival defects with fetal membranes". Archives of Ophthalmology 23 (1940): 522-525.

\section{Assets from publication with us}

- Prompt Acknowledgement after receiving the article

- Thorough Double blinded peer review

- Rapid Publication

- Issue of Publication Certificate

- High visibility of your Published work

Website: https://www.actascientific.com/

Submit Article: https://www.actascientific.com/submission.php Email us: editor@actascientific.com

Contact us: +919182824667 